\title{
Load-Induced Confinement Activates Diamond Lubrication by Water
}

\author{
G. Zilibotti, ${ }^{1}$ S. Corni, ${ }^{2}$ and M. C. Righi ${ }^{1,2, *}$ \\ ${ }^{1}$ Department of Physics, Informatics and Mathematics, University of Modena and Reggio Emilia, I-41125 Modena, Italy \\ ${ }^{2}$ Istituto Nanoscienze, CNR-Consiglio Nazionale delle Ricerche, I-41125 Modena, Italy
}

(Received 3 July 2013; published 2 October 2013)

\begin{abstract}
Tribochemical reactions are chemical processes, usually involving lubricant or environment molecules, activated at the interface between two solids in relative motion. They are difficult to be monitored in situ, which leaves a gap in the atomistic understanding required for their control. Here we report the real-time atomistic description of the tribochemical reactions occurring at the interface between two diamond films in relative motion, by means of large scale ab initio molecular dynamics. We show that the load-induced confinement is able to catalyze diamond passivation by water dissociative adsorption. Such passivation decreases the energy of the contacting surfaces and increases their electronic repulsion. At sufficiently high coverages, the latter prevents surface sealing, thus lowering friction. Our findings elucidate effects of the nanoscale confinement on reaction kinetics and surface thermodynamics, which are important for the design of new lubricants.
\end{abstract}

PACS numbers: 68.43.Bc, 62.50.-p, 68.35.Af, 68.35.Np

An important class of frictional phenomena occurring at the buried interface between two solids in relative motions, usually referred to as tribochemistry phenomena, is represented by the tribologically induced chemical modifications of the surfaces interacting with lubricants or other molecules present in the environment surrounding the sliding media [1]. These modifications can dramatically change the adhesion and friction of the materials in contact. Therefore, it is highly desirable to understand how they take place at the microscopic level. Our knowledge of the reaction kinetics and mechanisms at the open surface is not sufficient for this aim, since the tribological conditions can alter the rules of the game. These conditions include the dissipation of frictional energy, molecular confinement, shear strains, and high pressures. Usually, the reaction rate is observed to increase, a phenomenon referred to as "mechanical catalysis" in the field of mechanochemistry, where chemical and materials syntheses are achieved with the application of mechanical forces, as by grinding in ball mills [2,3]. Mechanosynthesis has seen a huge upsurge of interest in recent years driven by the quest of solvent-free reactivity in a number of areas [4]. However, fundamental understanding of many aspects of mechanochemistry as well as tribochemistry remains scarce due to the difficulties in directly probing the interface: the a posteriori characterization of the reaction products gives no insights into the pathways that lead to them [5,6]. Improving our understanding of the kinetics and mechanisms of tribochemical reactions is important to find solutions for the problems of wear and friction in many applications, such as the design of new, environmental friendly lubricants and represents an intriguing basic science problem by itself.

The use of high wear resistant and low friction coatings, such as diamond and diamondlike carbon, has been identified as the most popular strategy for green lubrication
[7,8]. Smooth, highly $s p^{3}$-bonded, nearly hydrogen-free carbon films, like ultra or nanocrystalline diamond (UNCD/NCD) and tetrahedral amorphous carbon (ta-C), possess mechanical properties, such as hardness, similar to those of single crystal diamond and can exhibit extremely low friction and wear in the absence of any liquid lubricant $[9,10]$. These materials are being used in many applications ranging from MEMS/NEMS [11] to engine components [12]. However, their widespread use has been limited by the difficulty in controlling the tribological properties in different environments: poor performances are obtained in vacuum or dry atmospheres compared to environments containing passivating species like hydrogen, water molecules or -OH containing lubricants [13-20]. On the basis of surface spectroscopy performed after the tribological test in humid environments, it has been pointed out that the key mechanism for ultralow friction in carbon films is surface passivation [17]. Results of first principles calculations are in line with this hypothesis: the calculated adhesion [21,22] and intrinsic resistance to sliding [19,23] of passivated diamond surfaces are very low, and become greater with the increase of the concentration of carbon dangling bonds (DBs) [24]. This latter result was also obtained by MD simulations of hydrogenated sliding surfaces [25-28]. On the basis of these experimental and theoretical clues, a tribochemical mechanism consisting in the balancing between (i) DBs formation by sliding and (ii) their passivation by adsorption of gaseous species, water in particular, has been hypothesized to explain diamond tribology $[17,20]$, but actually not yet directly observed. Here we elucidate such mechanisms and also provide information on the kinetics of tribochemical reactions. Beside being strategic for tribological applications, water at diamond interfaces represents, in fact, a well-defined system, where fundamental aspects of tribochemistry can be investigated. 
We perform ab initio molecular dynamics (MD) based on the Car Parrinello method [29]. In this way we fully take into account the electronic structures of the surfaces and molecules. This is important for an accurate description not only of chemical reactions, but also of water under confinement in general [30]. We considered five different systems constituted by partially hydrogenated and clean diamond surfaces interacting with interfacial water molecules in different amounts. The simulations covered time intervals ranging from 5 to 12 ps. The water molecules were initially positioned in the configuration that we have identified as the most stable for physisorption both on the clean and the partially saturated surfaces [31]. Deuterium mass is assigned to hydrogen atoms to allow longer time steps and to yield numerically more stable simulations. Dynamical events involving $\mathrm{H}$ atoms may be slowed by this choice. Yet, we observe a large variety of processes involving $\mathrm{H}$ atoms within the simulated time scale, showing that this choice does not prevent obtaining useful and novel insights. The large number of atoms included in our system (up to 478) and the simulated time intervals render our simulations computationally very demanding, in particular they required a vast amount of resources (about $400 \mathrm{k}$ cpu hours on highly parallel supercomputers), which points out the importance and the complexity of the current work. Further computational details are available as Supplemental Material [32].

Tribochemical reactions at clean diamond interfaces.We consider the (001) surface of diamond, which presents a $(2 \times 1)$ reconstruction constituted of dimers where the carbon atoms form $\mathrm{C}=\mathrm{C}$ double bonds. The interface is composed by two self-mating surfaces identically oriented, where the alternating dimer rows and trenches give rise to atomic-scale roughness. A load of $10.3 \mathrm{GPa}$ is gradually applied [33] and the system temperature is kept constant at $300 \mathrm{~K}$. We first consider a clean (i.e., hydrogen-free) interface, then we introduce water molecules in two different amounts. The atomic trajectories are displayed in the Supplemental Material [32] (movies No. 1-3).

When two clean diamond films are brought into contact, covalent bonds are established across the interface and the two surfaces undergo a cold sealing in spite of the stable reconstruction initially present. In a previous study we have shown that $\mathrm{C}-\mathrm{C}$ bonds across the interface can be established for different relative lateral positions of the two clean surfaces. The most favorable one has an associated work of adhesion of $7.5 \mathrm{~J} / \mathrm{m}^{2}$ (corresponding to an energy gain of about $6 \mathrm{eV}$ per $(2 \times 1)$ cell due to surface sealing) [24].

The presence of water at very low interfacial coverage, $\theta=0.08(\theta$ is defined as the number of molecules per interfacial dimer), cannot prevent the formation of C-C bonds across the interface: the molecules start their dissociation in $-\mathrm{H}$ and $-\mathrm{OH}$ fragments while the surfaces approach each other. When the space at the interface gets too small, the $\mathrm{OH}$ groups undergo a further dissociation and elemental oxygen and hydrogen remain embedded in the diamond matrix that is formed upon surface sealing (Fig. 1).

The situation is completely altered at higher water coverage, $\theta=0.5$ : while the surfaces get close, the molecules gather at the interfacial valleys, then processes of dissociative chemisorption take place and the thickness of the interfacial water film progressively reduces until all the molecules become dissociated. After that, the passivated surfaces remain separated in spite of the presence of a residual DBs concentration of 50\% and of the high applied pressure (Fig. 2). This effect is due to the Pauli repulsion between the adsorbed fragments on the opposite sides of the interface. The important consequence is that the two surfaces cannot reach a small enough separation to allow for the formation of C-C bonds across the interface, with a large reduction of frictional forces due to adhesion. We have, in fact, shown that the shear strength of diamond interfaces dramatically increases when $\mathrm{C}-\mathrm{C}$ bonds are established across the interface [e.g., from tens (hundreds) of MPa for fully (partially) passivated interfaces where $\mathrm{C}-\mathrm{C}$ bonds are not present, to tens of GPa where chemical bonds are established across the interface] [19,24].

The observation of surface passivation through dissociative water adsorption in the $a b$ initio simulation is in agreement with the outcomes of combined friction measurements and spectroscopic analysis performed in the presence of water vapor [14,18-20,34]. The energetic competition between cold sealing and surface passivation via water adsorption was analyzed previously [35], leading to the conclusion that high gas pressures can tip the balance toward surface passivation. The confinement created by the high applied load can constitute the driving force for the observed process and also account for the reaction rates observed in our simulations, which appear accelerated with respect to what is expected on the basis of the calculated

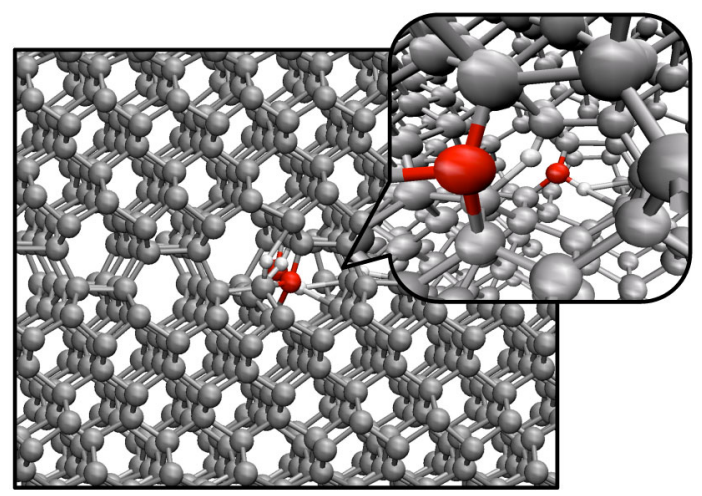

FIG. 1 (color online). Configuration assumed by the clean diamond interface at the end of the molecular dynamics simulation under $10.3 \mathrm{GPa}$ pressure and in the presence of a water coverage of 0.08 molecules per carbon dimer (corresponding to 2 water molecules per supercell). The full simulation movie is available as Supplemental Material [32]. 


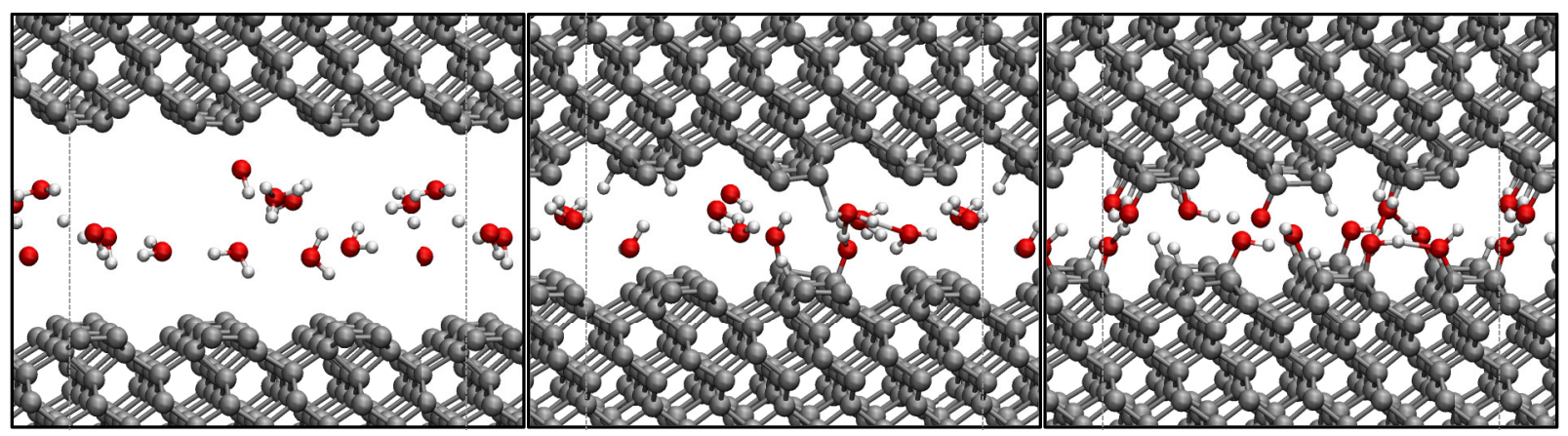

FIG. 2 (color online). Snapshots (subsequent in time from left to right) acquired during the molecular dynamics simulation of a clean diamond interface under $10.3 \mathrm{GPa}$ pressure and 0.5 water molecules per carbon dimer confined at the interface (corresponding to 12 water molecules per supercell). The width of each snapshot is slightly larger than the lateral supercell extension, the latter is indicated by dashed lines. The full simulation movie is available as Supplemental Material [32].

energy barriers for water dissociation on the clean surface [31]. We have, in fact, seen that both at low and medium coverages, the dissociation of all the molecules occurs almost immediately (i.e., on 0.5 ps time scale) after the load is increased to $10.3 \mathrm{GPa}$. To further investigate the role of the load, we repeat the simulation at $\theta=0.5$ coverage, now keeping the load constant at the lower value of $2.5 \mathrm{GPa}$ until the end of the simulation. As it can be seen from the atomic trajectories (movie No. 4 in the Supplemental Material [32]) and from the number of adsorption events as a function of time reported in Fig. 3, the processes of molecular dissociation take place throughout the 12 ps of the ab initio simulation, and few water molecules still remain undissociated at its end. The average interfacial separation for this second simulation is $26 \%$ larger than in the simulation at higher load, suggesting that upon interface compression at high loads, the volume per molecule may become too small for the molecular phase to remain stable. This is consistent with the results of previous classical [36] and ab initio [37] MD simulations of water under pressure.

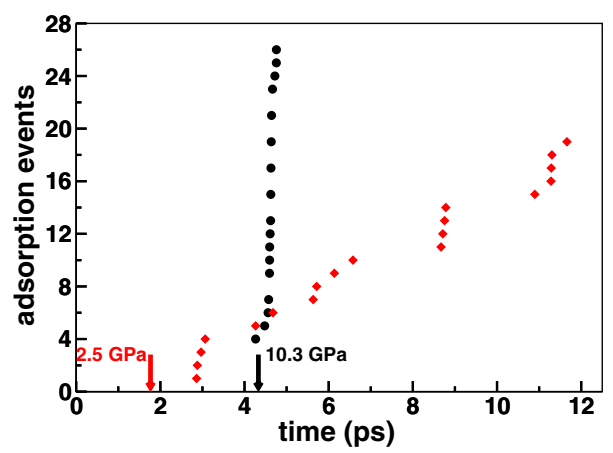

FIG. 3 (color online). Number of water fragments $(\mathrm{H}$ and $\mathrm{OH})$ adsorbed at the clean diamond interface during the dynamic simulations at different applied loads (movies No. 3 and 4 in the Supplemental Material [32]). The number is incremented whenever a water fragment $(\mathrm{H}$ or $\mathrm{OH})$ attaches to one of the two facing surfaces.
Tribochemical reactions at partially hydrogenated diamond interfaces.-Hydrogen surface termination and bulk content can highly influence the tribological properties of diamondlike carbon and diamond [19,38-41]. When hydrogenated, the $\mathrm{C}(001)$ surface maintains the $(2 \times 1)$ periodicity, with the carbon atoms involved in dimers forming single $\mathrm{C}-\mathrm{C}$ bonds and polar $\mathrm{CH}$ bonds. We study the dynamics of a partially hydrogenated interface, containing a DB density of $17 \%$, under the effects of an applied pressure of about $19.3 \mathrm{GPa}$ and a lateral force that produces the relative motion of the two surfaces. The atomic trajectories are displayed in movie No. 5 of the Supplemental Material [32]. During sliding, the distance between the two surfaces does not remain constant due to the atomic scale roughness: the external applied vertical force is instantaneously counterbalanced by the interfacial repulsion; therefore, the separation increases whenever the $\mathrm{CH}$ bonds of the top surface move over the $\mathrm{CH}$ bonds of the underlying surface. We have not detected the formation of any $\mathrm{C}-\mathrm{C}$ bond across the interface during the simulation, which lasted 9 ps. Rather, we have identified numerous events of $\mathrm{H}$ diffusion across the interface, always occurring at the minimum surface separation (one of such $\mathrm{H}$ diffusion events is represented in Fig. 4).

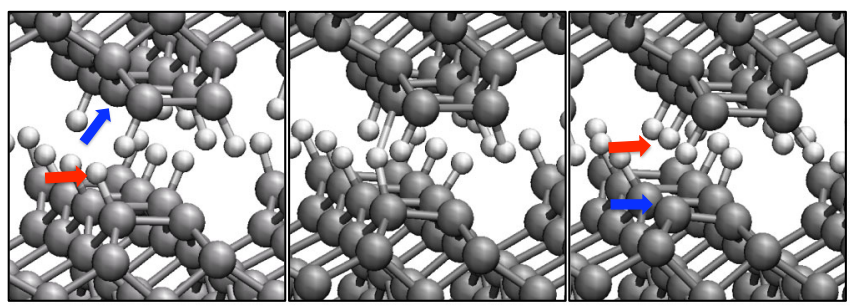

FIG. 4 (color online). Process of hydrogen diffusion across the interface observed during sliding of hydrogenated diamond interface under 19.3 GPa load (the full dynamics is available as a movie in the Supplemental Material [32]). The arrows indicate the $\mathrm{H}$ atom and the carbon dangling bond involved in the diffusion process. 
In particular, we observe that $\mathrm{H}$ diffusion causes the number of partially saturated dimers to decrease during the simulation and favor the formation either of fully saturated, or clean dimers. To rationalize the result, we calculate the energy cost for $\mathrm{H}$ removal either from a partially, or fully hydrogenated dimer, taking into account different terminations of the neighboring dimers. We find that $\mathrm{H}$ detachment from a partially saturated dimer requires $35 \%$ less energy than that required to detach a $\mathrm{H}$ atom from a fully hydrogenated dimer ( $1.44 \mathrm{eV}$ versus $2.23 \mathrm{eV}$, averaging on the different considered terminations of the neighboring dimers) because the product of the first reaction involves a clean dimer which is stabilized by $\mathrm{C}=\mathrm{C}$ double bonding [42]. For the same reason, the detached $\mathrm{H}$ prefers to fully saturate an already partially saturated dimer than to disrupt the stability of a clean $\mathrm{C}=\mathrm{C}$ dimer. This analysis elucidates the driving force for the process of $\mathrm{H}$ diffusion across the interface, brought out by our simulations. Experiments have not detected this phenomenon yet; possibly an experiment where the two sliding surfaces are terminated one with hydrogen and the other with deuterium may be able to detect the transfer a posteriori by spectroscopic analysis. The exchange of $\mathrm{H}$ atoms can play a role in energy dissipation. In fact, the $\mathrm{H}$ that transfers from one surface to the other is transiently creating a three-center two-electron chemical bond between the two surfaces (Fig. 4). Furthermore, the process of $\mathrm{H}$ diffusion observed in our simulations leads us to suppose that the number of DBs can increase, a process that has been hypothesized by experiments [20,41], but cannot happen in our simulations where the number of atoms is locally conserved due to periodic boundary conditions.

We consider the presence of water at low coverage, $\theta=0.08$, confined within the above described partially hydrogenated interface in sliding conditions, with $10.3 \mathrm{GPa}$ applied load. During the simulation (movie No. 6 in the Supplemental Material [32]) we observe the almost instantaneous dissociation of the water molecules, whose fragments saturate part of the DBs present at the interface. Both keto and hydroxyl groups are formed and transform one into the other during the simulation. A process of $\mathrm{COH}$ transformation into the less cumbersome $\mathrm{CO}$ group is shown in Fig. 5. We can see that it occurs when an incoming saturated dimer belonging to the above surface reduces locally the interfacial space. To understand this behavior, we calculate the reaction energy associated to the process of hydroxyl transformation into a keto group on a partially hydrogenated diamond surface. The process is exothermic by $0.33 \mathrm{eV}$ if $\mathrm{H}$ detachment from the $\mathrm{OH}$ group complete the passivation of a dimer. On the contrary, it is endothermic by $0.49 \mathrm{eV}$ if a partially passivated dimer is created upon $\mathrm{H}$ transfer from $\mathrm{OH}$ to a clean dimer. Interestingly, we find that the presence of an hydrogenated countersurface under $10.3 \mathrm{GPa}$ pressure is able to change these reaction energies. In particular, the energy gain in the

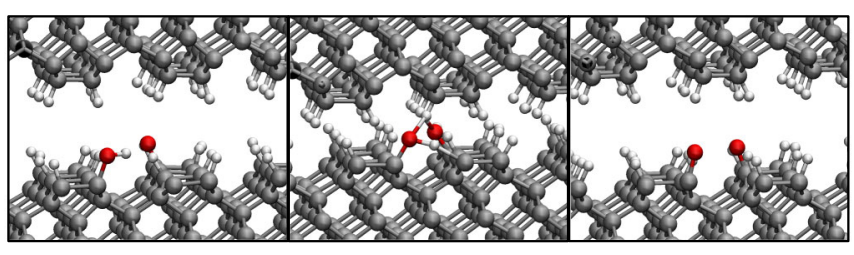

FIG. 5 (color online). Process of $\mathrm{COH}$ transformation into $\mathrm{CO}$ illustrated trough MD snapshots, observed after water dissociative adsorption at a partially hydrogenated diamond interface in sliding conditions. The movie of the whole simulation is presented as Supplemental Material [32].

first reaction increases to $0.72 \mathrm{eV}$ and the energy cost in the second reaction decreases to $0.23 \mathrm{eV}$. This indicates that the confinement condition reduces the stability of the $\mathrm{COH}$ group more than that of the $\mathrm{CO}$ one, most likely due to the higher steric hindrance of the former adsorbate (see the details of the calculation in the Supplemental Material [32]).

Our results can be compared with the available experiments of diamond-on-diamond tribochemistry. In particular, a recent study which correlates the surface chemical modifications with the tribological performances of selfmated UNCD as a function of the relative humidity and load, shows that the conditions to achieve low friction and wear are sufficiently high relative humidity (RH) and/or sufficiently low loads. The spectroscopic analysis revealed that worn regions present a higher concentration of oxygen than the unworn ones, indicating that when the original hydride termination is not maintained, the surfaces are passivated by dissociated water molecules [20]. These findings are in very good agreement with our simulations, which show that water molecules compressed between $\mathrm{H}$-depleted diamond surfaces promptly dissociate and passivate the surfaces. The Pauli repulsion between the soformed fully saturated bonds, as $\mathrm{CH}$ and $\mathrm{COH}$, can stand the applied load and prevent the two facing surfaces from reaching distances in the range of the $\mathrm{C}-\mathrm{C}$ interaction. However, a high enough concentration of water molecules is necessary to achieve this condition: our simulations show that surface sealing cannot be avoided at low water coverage, in agreement with the measured increase of friction and wear at low RH levels. The a priori passivation, as hydrogenation, is an efficient method to reduce the adhesion and favor the relative surface sliding, as observed in nanotribological experiments on UNCD after exposure to atomic hydrogen [40]. $\mathrm{H}$ diffusion occurring during sliding of partially hydrogenated surfaces is also predicted.

Our simulations show that the tribological conditions alter the surface kinetics and thermodynamics. We find that the relative stability of different surface terminations under confinement is influenced by factors, such as the steric hindrance, that are irrelevant at the open surface. Moreover, we demonstrate that confinement under pressure is the driving force for molecular dissociation and 
reaction rates are functions of load. In particular, we found that the higher the load, the more effective the surface passivation by water dissociation is, which in turn leads to a reduction of friction. Therefore, our theoretical findings offer a possible explanation for a recent, counterintuitive, experimental result: at high relative humidity $(50 \%)$ the friction coefficient for ultrananocrystalline diamond and tetrahedral amorphous carbon is actually lower for the higher load [20]. No such behavior is seen at low relative humidity. While we cannot rule out that other effects, on larger spatial scales, determine this behavior [20], the molecular mechanism disclosed by our simulations certainly prompts further experimental investigations.

We thank the DEISA Consortium, cofunded through the EU FP6 Project No. RI-031513 and the FP7 Project No. RI-222919, for support within the DEISA Extreme Computing Initiative. Professor M. Ferrario is gratefully acknowledged for useful discussions. Dr. C. Cavazzoni is gratefully acknowledged for computational support.

*mcrighi@unimore.it

[1] S. Hsu, J. Zhang, and Z. Yin, Tribol. Lett. 13, 131 (2002).

[2] S. L. James, C. J. Adams, C. Bolm, D. Braga, P. Collier, T. Friscic, F. Grepioni, K. D. M. Harris, G. Hyett, W. Jones et al., Chem. Soc. Rev. 41, 413 (2011).

[3] T. Friscic, I. Halasz, P. J. Beldon, A. M. Belenguer, F. Adams, S. A. Kimber, V. Honkimaki, and R.E. Dinnebier, Nat. Chem. 5, 66 (2012).

[4] K. D. M. Harris, Nat. Chem. 5, 12 (2012).

[5] J. Fineberg, Nat. Mater. 10, 3 (2011).

[6] W. G. Sawyer and K. J. Wahl, MRS Bull. 33, 1145 (2011).

[7] R. Hauert, Tribol. Int. 37, 991 (2004).

[8] A. Erdemir, Tribol. Int. 38, 249 (2005).

[9] A. Sumant, D. Grierson, J. Gerbi, J. Birrell, U. Lanke, O. Auciello, J. Carlisle, and R. Carpick, Adv. Mater. 17, 1039 (2005).

[10] O. Williams, A. Kriele, J. Hees, M. Wolfer, W. M. Sebert, and C. Nebel, Chem. Phys. Lett. 495, 84 (2010).

[11] A. V. Sumant, O. Auciello, R. W. Carpick, S. Srinivasan, and J.E. Butler, MRS Bull. 35, 281 (2011).

[12] S. C. Tung and H. Gao, Wear 255, 1276 (2003).

[13] M. N. Gardos and S.A. Gabelich, Tribol. Lett. 6, 103 (1999).

[14] S. Grillo and J. Field, Wear 254, 945 (2003).

[15] H. Kim, J. Lince, O. Eryilmaz, and A. Erdemir, Tribol. Lett. 21, 51 (2006).

[16] C. Matta, M. I. De Barros Bouchet, T. Le-Mogne, B. Vachet, J. M. Martin, and T. Sagawa, Lubr. Sci. 20, 137 (2008).

[17] A. R. Konicek, D. S. Grierson, P. U. P. A. Gilbert, W. G. Sawyer, A. V. Sumant, and R. W. Carpick, Phys. Rev. Lett. 100, 235502 (2008).
[18] N. Kumar, N. Sharma, S. Dash, C. Popov, W. Kulisch, J. Reithmaier, G. Favaro, A. Tyagi, and B. Raj, Tribol. Int. 44, 2042 (2011).

[19] M.-I. De Barros Bouchet, G. Zilibotti, C. Matta, M. C. Righi, L. Vandenbulcke, B. Vacher, and J.-M. Martin, J. Phys. Chem. C 116, 6966 (2012).

[20] A. R. Konicek, D. S. Grierson, A. V. Sumant, T. A. Friedmann, J. P. Sullivan, P. U. P. A. Gilbert, W. G. Sawyer, and R. W. Carpick, Phys. Rev. B 85, 155448 (2012).

[21] S. Dag and S. Ciraci, Phys. Rev. B 70, 241401 (2004).

[22] Y. Qi, E. Konca, and A. T. Alpas, Surf. Sci. 600, 2955 (2006).

[23] G. Zilibotti, M. Righi, and M. Ferrario, Phys. Rev. B 79, 075420 (2009).

[24] G. Zilibotti and M. C. Righi, Langmuir 27, 6862 (2011).

[25] J. Harrison, D. Brenner, C. White, and R. Colton, Thin Solid Films 206, 213 (1991).

[26] G. T. Gao, P. T. Mikulski, and J. A. Harrison, J. Am. Chem. Soc. 124, 7202 (2002).

[27] Y. Mo, M. H. Müser, and I. Szlufarska, Phys. Rev. B 80, 155438 (2009).

[28] K. Hayashi, K. Tezuka, N. Ozawa, T. Shimazaki, K. Adachi, and M. Kubo, J. Phys. Chem. C 115, 22981 (2011).

[29] R. Car and M. Parrinello, Phys. Rev. Lett. 55, 2471 (1985).

[30] M. Sharma, D. Donadio, E. Schwegler, and G. Galli, Nano Lett. 8, 2959 (2008).

[31] O. Manelli, S. Corni, and M. C. Righi, J. Phys. Chem. C 114, 7045 (2010)

[32] See Supplemental Material at http://link.aps.org/ supplemental/10.1103/PhysRevLett.111.146101 for the atom trajectories recorded during the MD simulations, additional computational details, and the description of the first-principles calculations described in the text.

[33] A pressure of $0.5 \mathrm{GPa}$ is applied $0.48 \mathrm{ps}$ after the beginning of the simulation. The pressure is then raised to $2.5 \mathrm{GPa}$ at $t=1.8 \mathrm{ps}$ and finally to $10.3 \mathrm{GPa}$ at $t=$ 4.3 ps. This last value is kept constant during the rest of the simulation.

[34] J. Andersson, R. A. Erck, and A. Erdemir, Surf. Coat. Technol. 163-164, 535 (2003).

[35] H. Guo and Y. Qi, Model. Simul. Mater. Sci. Eng. 18, 034008 (2010).

[36] M. Paliy, O. M. Braun, and S. Consta, J. Phys. Chem. C 116, 8932 (2012).

[37] C. Cavazzoni, G. L. Chiarotti, S. Scandolo, E. Tosatti, M. Bernasconi, and M. Parrinello, Science 283, 44 (1999).

[38] C. Donnet, J. Fontaine, A. Grill, and T. Le Mogne, Tribol. Lett. 9, 137 (2001).

[39] J. Fontaine, C. Donnet, A. Grill, and T. LeMogne, Surf. Coat. Technol. 146-147, 286 (2001).

[40] A. V. Sumant, D. S. Grierson, J. E. Gerbi, J. A. Carlisle, O. Auciello, and R. W. Carpick, Phys. Rev. B 76, 235429 (2007).

[41] A. M'ndange-Pfupfu, J. Ciston, O. Eryilmaz, A. Erdemir, and L. Marks, Tribol. Lett. 49, 351 (2012).

[42] G. Zilibotti, S. Corni, and M. C. Righi, Phys. Rev. B 85, 033406 (2012). 